\title{
Connected Mobility Aids: Supporting Physically Impaired Traffic Participants with Vehicle-to-X Communication
}

\author{
Stefan Diewald \\ Distributed Multimodal Information Processing Group \\ Technische Universität München, Munich, Germany \\ stefan.diewald@tum.de
}

\author{
Patrick Lindemann, Matthias Kranz \\ Lehrstuhl für Informatik mit Schwerpunkt Eingebettete Systeme \\ Universität Passau, Passau, Germany \\ patrick.lindemann@uni-passau.de,matthias.kranz@uni-passau.de
}

\begin{abstract}
Due to population ageing, the number of people that depend on mobility aids, such as walking frames, mobility scooters, or wheel chairs, will increase steadily over the next decades. In order to support the physical impaired outside traffic participants, barrier-free navigation and trip planning solutions have been developed. In this work, we go beyond these approaches and investigate potential benefits arising from the integration of mobility aids in vehicle-to-x communication. We argue that this can lead to increased safety for all traffic participants as well as to a more efficient mobility for the physically impaired people as individual requirements can be met.
\end{abstract}

\section{INTRODUCTION AND BACKGROUND}

Independent mobility is a key aspect for self-determined living and social inclusion. However, with the demographic change leading towards population ageing, more and more people have physical impairments and are dependent on mobility aids; and the number will rise steadily over the next decades. For people with mobility aids, normal road traffic can already present an insurmountable barrier. A major problem is the negotiation of height differences (e.g., steps or curbs). In situations with other traffic participants, the significantly slower speed of mobility aid users in comparison to average pedestrians can cause dangerous situations. Both aspects make users of mobility aids more vulnerable in road traffic, as they need more time to cross streets and have to use the street in case of inadequate sidewalks. Together with the uncertainty of not knowing where they can go safely, users may avoid certain traffic situation which can heavily limit their mobility.

In a 2012 study on outdoor built environment barriers among people with mobility disabilities [1], Rosenberg et al. identified the themes that negatively affect the people's individual mobility. The main mobility barriers from this study are summarized in Table I. In many cases, these barriers can be overcome by providing real-time information or exchanging messages with other traffic participants. For that reason, we analyze the use of vehicle-to-X (V2X) communication in order to provide support in daily traffic for users of mobility aids.

\section{Potential ApPlications}

The potential applications are summarized in Table I. The themes are based on the summary of common barriers for users of mobility aids [1]. The user equipment can be a commercial off-the-shelf mobile device (smartphone or tablet PC) with V2X communication capabilities [2]. For use cases T1 (topologybased navigation) and T2 (real-time operational information),

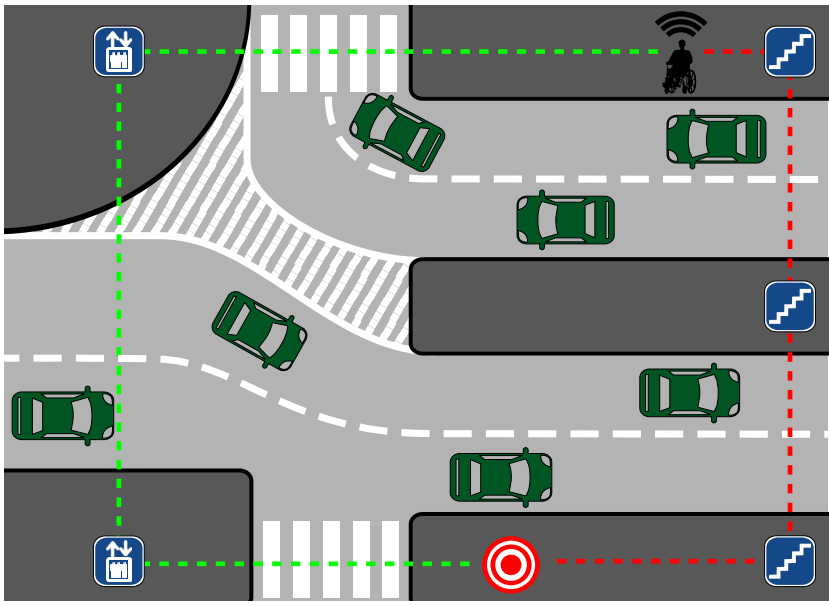

Fig. 1. T1 Topology-based navigation \& T2 real-time operational information. The topology description sent out by the roadside units via V2I communication could include barrier-free navigation information with real time data, such as the state of elevators. This could help users with mobility impairments to choose the optimal route.

data about the topology of intersections and surrounding sidewalks has to be broadcast via vehicle-to-infrastructure (V2I) communication. The description should also contain positions of pedestrian crossings and other auxiliary means for crossing the street (e.g., elevators to underground crossings). In addition, the current state of these auxiliary means should be included. The V2I data can then be combined with navigational information on the mobile device for providing enhanced routes. An example is depicted in Figure 1.

Themes T3 (crossing at traffic lights) and T4 (crossing the street) require the user equipment to transmit data. This can be done in a message that contains the current position, heading, and speed of the pedestrian. When a vehicle receives the data of a mobility aid user, it can present an appropriate warning to the driver. The same data can be evaluated by traffic lights. In case a user with mobility aid is crossing at the traffic light, it can automatically determine whether the crossing time needs to be prolonged. Example scenarios are depicted in Fig. 2 and Fig. 3.

\section{OPEN ISSUES AND FUTURE WORK}

As pedestrians in general are currently not included in V2X scenarios, a new message type needs to be introduced and standardized. The message would be similar to the cooperative awareness message (CAM) for vehicles, quasi a "pedestrian 
TABLE I. SELECTED BARRIERS FROM THE ANALYSIS OF ROSENBERG ET AL. [1] AND OUR IDENTIFIED V2X COMMUNICATION-BASED FACILITATOR APPROACHES. THE USER EQUIPMENT CAN BE THE USER'S MOBILE DEVICE WITH SUITABLE RADIO UNIT AND SOFTWARE.

\begin{tabular}{|c|c|c|}
\hline Theme & Barriers & V2X communication-based facilitator approach \\
\hline T1 Topography & $\begin{array}{l}\text { Lacking curb ramps, lack of adequate sidewalks, } \\
\text { lack of crosswalks (including barrier-free under- } \\
\text { passes and overpasses) }\end{array}$ & $\begin{array}{l}\text { V2I at intersections: provide a topological overview around intersection including curb ramps, } \\
\text { sidewalk sizes and crosswalk positions. Navigation application can evaluate data and refine } \\
\text { navigation instructions. }\end{array}$ \\
\hline $\begin{array}{l}\text { T2 Real-time operational } \\
\text { information }\end{array}$ & $\begin{array}{l}\text { Obstructed sidewalks, inoperative pedestrian } \\
\text { lights, inoperative elevators/escalators, inoperative } \\
\text { lighting }\end{array}$ & $\begin{array}{l}\text { V2I at intersections and crosswalks: provide real-time information on the state of ways and } \\
\text { technical equipment relevant for pedestrians, and list alternatives when out of order. Navigation } \\
\text { application can evaluate data and refine navigation instructions. }\end{array}$ \\
\hline T3 Crossing at traffic lights & Unknown crossing time, crossing time too short & $\begin{array}{l}\text { V2I at traffic lights: provide signal phase and timing (SPaT) information for pedestrian crossings. } \\
\text { User equipment can situationally prolong the crossing time. }\end{array}$ \\
\hline T4 Crossing the road & $\begin{array}{l}\text { Fast speed of car traffic, crossing at poorly } \\
\text { visible/obstructed locations, busy street without } \\
\text { nearby crosswalks }\end{array}$ & $\begin{array}{l}\text { V2X between crossing person and approaching traffic participants: provide exact position where } \\
\text { user is crossing or where user expresses intention to cross street }\end{array}$ \\
\hline
\end{tabular}

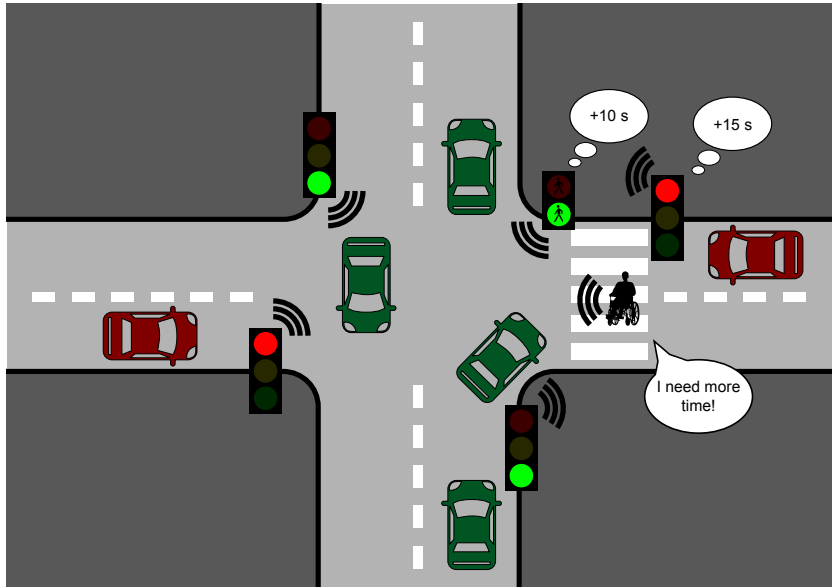

Fig. 2. T3 Flexible traffic light circuits: Users with mobility aids often need more time when crossing a street. By communicating with the traffic lights via V2I communication, the crossing time could be automatically prolonged.

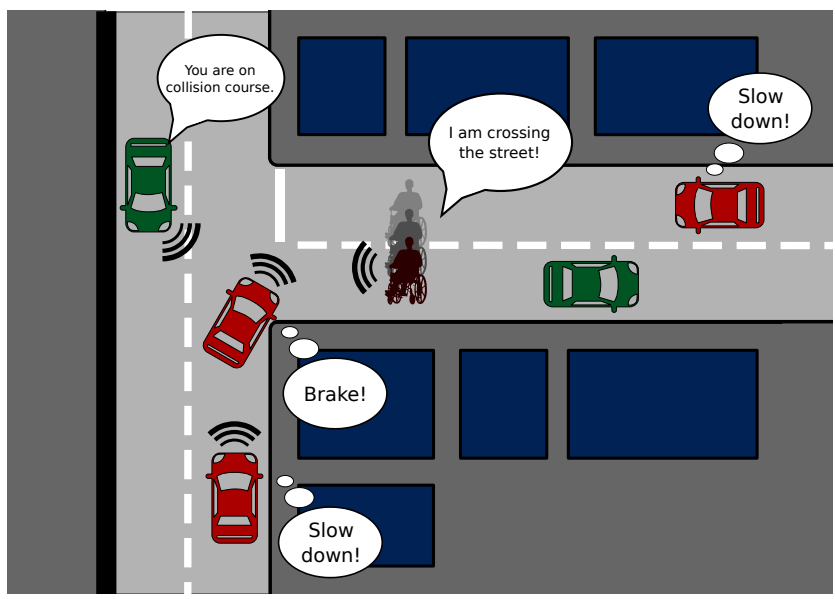

Fig. 3. T4 Crossing a street: In case of blocked sidewalks or due to lack of crosswalks, sometimes crossing the street at poorly visible locations is necessary. Via V2X communication, other traffic participants could be warned and informed that the crossing may take longer.

awareness message" (PAM). This message should be sent out as soon the pedestrian starts crossing the street or is using the street. Special message fields could be used to indicate whether the pedestrian is using a mobility aid. This information could be evaluated by the infrastructure in order to, e.g., prolong the crossing time at traffic lights (use case T3). For delivering realtime information on elevators or sidewalk states, an enhanced topology description format would be necessary [3].
Since the user equipment needs to be powered by battery, a low power radio system is necessary. It would be either possible to equip the devices with IEEE 802.11p-compatible radio units, or switch to an existing low power standard. For example, Bluetooth low energy (also known as Bluetooth Smart) would be an appropriate short range wireless communication standard that offers low latency. As with V2X applications in general, a certain penetration rate is necessary in order to create a benefit for the users. When realizing the services via the users' mobile devices, the adoption is dependent on whether new hardware is necessary or not. If new hardware is necessary and the technology is adopted by the large mobile handset manufacturers, a high penetration rate can be reached within one common mobile device life cycle, which is about 18 months ${ }^{1}$. Necessary infrastructure upgrades would accompany the upgrades that are necessary for V2I communication [4]. For use case T4 (crossing the road), the equipment rate of vehicles is also decisive. As the life cycle of vehicles is much longer, an adoption rate of more than $90 \%$ is expected 15 years after broad market introduction [5].

All in all, V2X communication has the potential to improve the mobility situation for physically impaired people by supporting them with accurate real-time information and informing other traffic participants about them. In addition, with higher grades of driving automation, vehicles can use the data received from crossing pedestrians to avoid potentially dangerous situations. Future work includes evaluating radio standards and defining appropriate message format.

\section{REFERENCES}

[1] D. E. Rosenberg, D. L. Huang, S. D. Simonovich, and B. Belza, "Outdoor Built Environment Barriers and Facilitators to Activity among Midlife and Older Adults with Mobility Disabilities," The Gerontologist, vol. 53, no. 2, pp. 268-279, Apr. 2013.

[2] S. Diewald, T. Leinmüller, B. Atanassow, L.-P. Breyer, and M. Kranz, "Mobile Device Integration and Interaction with V2X Communication," in 19th World Congress on Intelligent Transport Systems (ITS), Oct. 2012.

[3] S. Röglinger, "A Methodology for Testing Intersection Related Vehicle-2X Applications," Computer Networks, vol. 55, no. 14, pp. 3154-3168, Oct. 2011.

[4] H. Rakha and R. Kamalanathsharma, "Eco-Driving at Signalized Intersections Using V2I Communication," in Proceedings of the 14th International Conference on Intelligent Transportation Systems, ser. ITSC '11. IEEE, Oct. 2011, pp. 341-346.

[5] F. Bai and B. Krishnamachari, "Exploiting the Wisdom of the Crowd: Localized, Distributed Information-centric VANETs," Communications Magazine, vol. 48, no. 5, pp. 138-146, May 2010.

\footnotetext{
${ }^{1}$ http://www.nytimes.com/2011/02/26/business/26upgrade.html
} 